\title{
An Investigation on the Accuracy of Regional Ocean Models Through Field Trials
}

\author{
Ryan N. Smith, Jonathan Kelly, Kimia Nazarzadeh and Gaurav S. Sukhatme
}

\begin{abstract}
Recent efforts in mission planning for underwater vehicles have utilised predictive models to aid in navigation, optimal path planning and drive opportunistic sampling. Although these models provide information at a unprecedented resolutions and have proven to increase accuracy and effectiveness in multiple campaigns, most are deterministic in nature. Thus, predictions cannot be incorporated into probabilistic planning frameworks, nor do they provide any metric on the variance or confidence of the output variables. In this paper, we provide an initial investigation into determining the confidence of ocean model predictions based on the results of multiple field deployments of two autonomous underwater vehicles.

For multiple missions of two autonomous gliders conducted over a two-month period in 2011 , we compare actual vehicle executions to simulations of the same missions through the Regional Ocean Modeling System in an ocean region off the coast of southern California. This comparison provides a qualitative analysis of the current velocity predictions for areas within the selected deployment region. Ultimately, we present a spatial heat-map of the correlation between the ocean model predictions and the actual mission executions. Knowing where the model provides unreliable predictions can be incorporated into planners to increase the utility and application of the deterministic estimations.
\end{abstract}

\section{INTRODUCTION}

A key problem in the study of ocean science is the estimation of physical quantities, such as chlorophyll, dissolved oxygen, and temperature, across vast spatiotemporal expanses. The recent applications of satellite technology, autonomous marine vehicles, and sensors placed on drifting platforms have made it feasible to obtain a more complete view of these quantities. This, in turn, has lead to a significant benefit for biological science, physical oceanography, and climate monitoring by combining these data to develop predictive models and drive future sampling efforts. While this seems to imply that data are abundant, there is a serious impediment to our further understanding of many dynamic ocean processes. Gathered data, are often noisy and potentially unavailable across large areas and time periods, e.g., clouds obscuring a synoptic view from satellites. Even with all the advances, we are still only able to collect sparse data sets both spatially and temporally.

R.N. Smith and K. Nazarzadeh are with the School of Electrical Engineering and Computer Science, Queensland University of Technology, Brisbane, QLD, 4001 Australia. kimia.nazarzadeh@qut.edu.au, ryan.smith@qut.edu.au

J. Kelly is with the Robust Robotics Group, Computer Science and Artificial Intelligence Laboratory, Massachusetts Institute of Technology, Cambridge, MA 02142, USA. jskellyecsail.mit.edu

G. Sukhatme is with the Robotic Embedded Systems Laboratory, Department of Computer Science, University of Southern California, Los Angeles, CA 90089, USA. gaurav@usc.edu

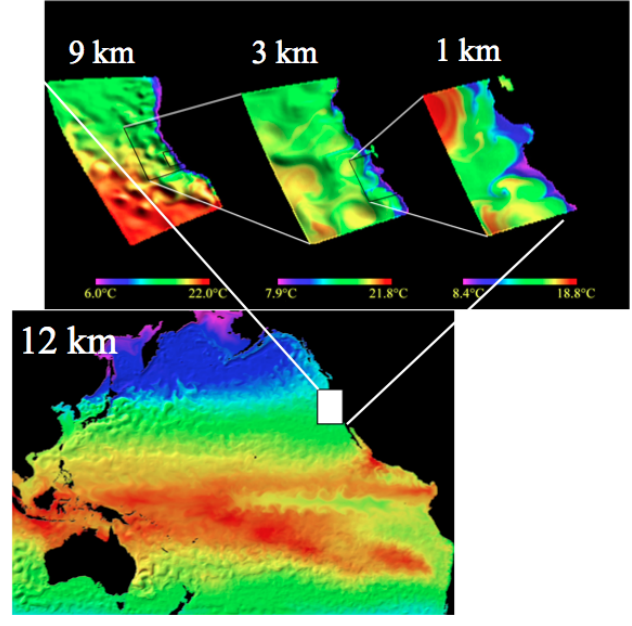

Fig. 1. An example output of a sea-surface temperature prediction for each of the available nested grids (resolutions of 12,9,3 and $1 \mathrm{~km}$ ) from the ROMS run by the Jet Propulsion Laboratory. Image courtesy of Yi Chao, Jet Propulsion Laboratory.

The complexity of oceanic processes and the chronic undersampling of the oceans inevitably leads to the necessity for models to fill the gap between observation, understanding, and prediction of these processes. Numerical models have been developed, and are configurable for a specific application or region of the world's oceans; with many instances now in a very mature state. For example, the Regional Ocean Modeling System (ROMS) has configurations for multiple locations along the western United States, including southern California and Monterey Bay (Fig. 1) [1], [2]; the Harvard Ocean Prediction System (HOPS) has been applied in multiple areas around the world [3][5]; and the Navy Coastal Ocean Model (NCOM ICON) Innovative Coastal-Ocean Observing Network is configured for the California coast and Monterey Bay [6]-[8]. There has also been extensive research for each of these examples to assimilate spatiotemporal data from multiple sources into these models to improve their overall predictive capabilities, see [9]-[13]. These models, along with the many others that exist, are able to predict measurements of current velocity, temperature, salinity, chlorophyll, and other physical and biological parameters in three spatial dimensions for times ranging from a few hours up to a few days. One drawback is that the predictions are generally deterministic in nature.

Autonomous Underwater Vehicles (AUVs) provide a nonintrusive, and a repeatable method for observing the oceans up close, and cost-effectively augmenting the data provided by existing methods. With existing AUVs having endurances 
ranging from a few days to months, a core challenge is predicting or deciding where and/or when to deploy these assets to maximise the information gain with respect to the process of interest in the ocean. This challenge has motivated the investigation into the utility of a priori measurements and/or predictions (priors) to solve path planning and asset allocation problems, e.g., [14]-[20]. The need for such prior information is motivated by: 1) robots have limited resources, 2) we need to plan robot motion, 3) we need to plan efficient robot motion when operating in an environment as complex as the ocean, and 4) effective ocean observation requires accurate spatiotemporal sampling - gathering data within or following a specific mass of water at/for a certain time.

Combining predictive models with the physics of the water column and particulate advection, chemistry of molecular diffusion, ecology of organism reproduction and survival, and bathymetric surveys, provides a significant advantage when trying to sample a moving patch of water. Here, waypointbased navigation strategies can lead sampling efforts astray. If a vehicle repeatedly tries to hit a prescribed waypoint, the interesting patch of water is gone. Thus, we need to utilise the priors to put the AUV in the right place at the right time to gather the important information. This requires an understanding on the accuracy and/or probability distribution of the output of model predictions. The complexity and expense of this task lies in both obtaining ground truth through field deployments (multiple robot deployments covering large areas over long amounts of time) and in extending existing models to provide variance metrics correlated to their predictions (computation time and resources).

To this end, this paper provides an initial investigation into the ground-truthing portion of the horizontal ocean current predictions of ROMS off the coast of southern California. We examine a set of experiments conducted by autonomous gliders in a localised region over a period of two months in 2011. The actual executions of the missions are compared with simulations of these missions that incorporate the ocean current predictions from ROMS prediction for the associated time period of the actual mission.

\section{BACKGROUND}

The motivation for this work stems from a desire to enable intelligent data collection of complex dynamics and processes that occur in a coastal ocean environments to further our understanding and prediction capabilities. Of particular interest is the formation and evolution of Harmful Algal Blooms (HABs) in the Southern California Bight (SCB); an oceanic region contained within $32^{\circ} \mathrm{N}$ to $34.5^{\circ} \mathrm{N}$ and $-117^{\circ}$ $\mathrm{E}$ to $-121^{\circ} \mathrm{E}$. This region is under continued study with the motivation to understand the connections between smallscale biophysical processes and large-scale events related to algal blooms, and especially those blooms composed of toxin producing species, commonly referred to as Harmful Algal Blooms (HABs) [21]-[24]. This long-term study utilises a team of autonomous gliders ( $\mathrm{He} \mathrm{Ha} \mathrm{Pe}$ and Rusalka), owned and operated by the USC CINAPS team [23], to sample along the San Pedro Shelf (see Fig. 2(b)). Missions

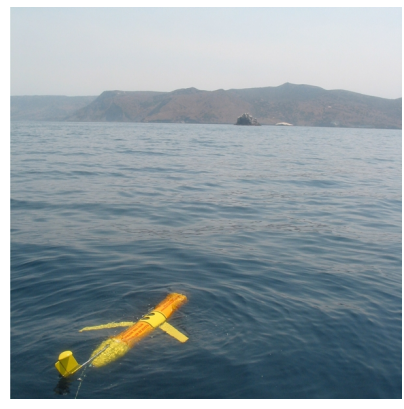

(a) A Slocum glider.

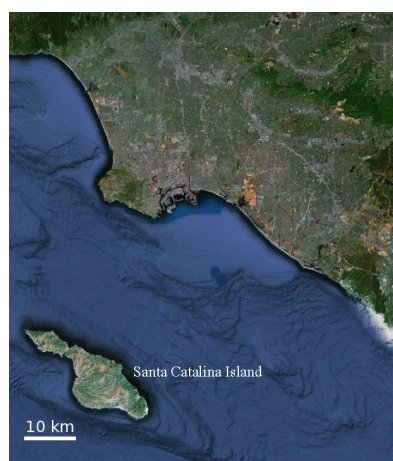

(b) Glider deployment region.
Fig. 2. (a) One of the two Slocum gliders owned and operated by the USC CINAPS [23]. The glider is preparing to start a mission just off the Northeast coast of Santa Catalina Island, CA. (b) The San Pedro Shelf contained within the SCB and the primary deployment region for CINAPS gliders.

executed by these vehicles comprise the field data examined within this paper. The Jet Propulsion Laboratory, California Institute of Technology, maintain a high-resolution, largescale regional ocean model for the SCB, and provided the model predictions used for our simulation comparisons [25].

The use of priors in the form of predictive ocean models has been shown to improve mission accuracy and execution during field trials [26], [27]. As advanced as these models have become, the predictions are still generally discrete and deterministic in nature. Specifically, there is no metric provided on the confidence or covariance of the predicted variable, and accurate interpolations of the discrete measurements requires sophisticated data processing algorithms to augment the predictions. The developers of both ROMS and HOPS have investigated producing ensemble forecasts for their model. This method perturbs initial and/or boundary conditions to produce a set of forecasts. This ensemble forecast provides a distribution of potential outcomes, however the sample size is often too small $(\sim 12)$ to provide a meaningful variance measure of the prediction. These ensembles can provide an accuracy estimation of the model output, however this is more related to the sensitivity of the numerical model to the inputs rather than the actual physical variability inherent within the domain of the model. The size of the ensemble forecast is limited only by computational power and time. However, as models provide the most accurate predictions in the times closest to observations and measurements, we cannot spend an excessive amount of time computing all the possible ensemble outcomes. So, we are faced with the compromise of working to produce the most accurate models utilising a minimal set of resources, or utilising additional resources to augment the computation of model reliability and variability. In current research, several assimilation techniques, e.g., Kalman filter-based ensemble forecasts [28], and variational schemes, e.g., 4DVar [29], are under investigation in the modeling community, with the goal to get the model output closer to the observed values.

The use of model predictions for planning AUV missions has been exploited in previous research efforts. A main focus 
of existing studies is computing energy optimal paths, as the majority of applications utilise propeller-driven, shortduration $(<24 \mathrm{hr})$ AUVs. The work in [30] implements a genetic algorithm to exploit time-varying currents for energy savings of an AUV. The spatial variability of currents is examined, and exploited with an $\mathrm{A}^{*}$ planner, in [14]. The authors in [17] examine the utility of models to help avoid land masses exposed by ebbing tides. And, the use of model predictions for flow in estuarine and riverine environments is presented in [16]. Although the methodologies, domains and applications are different, the underlying models used are all deterministic, providing a fixed value for the current at a specified location and time. Recent research in robotics is utilising probabilistic methods for planning and control, and has the potential for many applications in the marine domain, e.g., [31]-[33]. Hence, it is important to understand the probabilistic nature and/or variability of the priors that will best enable ocean sampling, e.g., predictive ocean models.

\section{A. Autonomous Underwater Vehicle}

The vehicle used in our field trials was a coastal (shallow) model of the Webb Slocum autonomous underwater glider [34] as shown in Fig. 2(a). This is a 1.5 m (length) by 21.3 $\mathrm{cm}$ (diameter), cylindrical vehicle designed for long-term ( 1 month) ocean observation [35], [36]. An autonomous glider flies through the water utilising a variable buoyancy system. Wings convert the buoyancy-dependent vertical motion into forward velocity. Inflection points occur at userdefined depths and altitudes. A glider navigates by deadreckoning between prescribed waypoints with a sequence of dives and climbs, forming a vertical sawtooth (yo-yo) pattern. When navigating to a new waypoint, the present location $L$ of the vehicle is compared to the next prescribed waypoint in the mission file $\left(W_{i}\right)$, and a bearing and range are computed for execution of the next segment of the mission. The geographical location at the extent of the computed bearing and range from $L$ is the aiming point $A_{i}$. The vehicle deadreckons with the computed bearing and range towards $A_{i}$, with the intent of surfacing at $W_{i}$. The computed bearing is not altered, and the glider must surface to make any corrections or modifications to its dive plan. When the glider determines that it has travelled the requested range at the specified bearing (based on speed over ground estimation from the previous dive), it surfaces and acquires a GPS fix at point $S$. If the vehicle surfaces within a given range of $W_{i}$, the waypoint is determined to be achieved. Positional error between the actual surfacing location and $W_{i}$ is computed, and is fully attributed to environmental disturbances, i.e., ocean currents. A depth-averaged current vector is computed, and this is factored in when computing the range and bearing to $W_{i+1}$. Hence, $A_{i}$ is in general not in the same physical location as $W_{i}$, and rarely does the glider ever surface at $W_{i}$.

Given their persistence, gliders are useful for understanding physical forcing components acting on a regional scale and determining long-term variability [23], [37]. Methods to increase the navigational accuracy of gliders via model predictions has been studied by the authors in [18], [26].

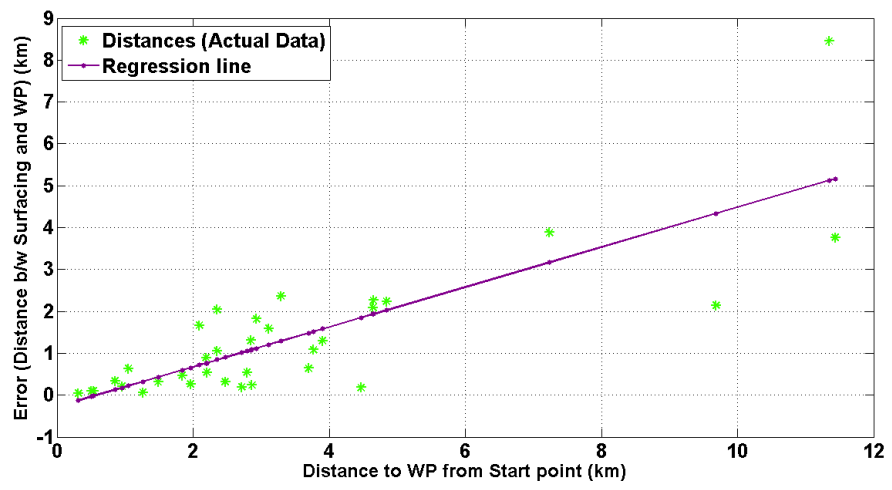

Fig. 3. A plot of the absolute surfacing error (in $\mathrm{km}$ ) versus the length of the prescribed trajectory for the actual field trials.

These works motivate further research in high-level planning methods to increase the ability of gliders to perform accurate spatiotemporal sampling over large scales, and to extend the utility of priors in planning for AUVs.

\section{B. Regional Ocean Modeling System}

The Regional Ocean Model System (ROMS) is maintained by the Jet Propulsion Laboratory, California Institute of Technology and is a split-explicit, free-surface, topographyfollowing-coordinate oceanic model. The model output has three nested horizontal resolutions covering the U.S. west coastal ocean $(15 \mathrm{~km})$, the southern California coastal ocean $(5 \mathrm{~km})$ and the SCB $(2.2 \mathrm{~km})$. The three nested ROMS domains (see Fig. 1) are coupled online and run simultaneously exchanging boundary conditions at every time step. The vertical resolution is non-uniform, providing data at depths ranging from 0 to $2000 \mathrm{~m}$. ROMS provides hindcasts, nowcasts and hourly forecasts (up to 72 hours) for the SCB, [1], [25].

\section{RESULTS}

\section{A. FIELD EXPERIMENTS}

The experiments in this study were conducted between 14 July and 3 August, 2011. During this time, two gliders were deployed in the SCB, shown in Fig. 2(b), executing predefined sampling missions, yo-yoing between 2 and $80 \mathrm{~m}$. Further details of the standard missions can be found in [38], [39]. From all of the surfacing locations during the mission executions, we selected those where the subsequent surfacing hit a waypoint. Thus, we selected the dives for which we knew the start location at the surface $L$, the desired waypoint $W_{i}$, the aiming point $A_{i}$, and the actual surfacing location $S$ of the vehicle for each segment examined. These selected mission segments comprise 34 surfacing events over a span of 21 days, with which to compare to simulated missions.

For each of the 34 missions examined, Fig. 3 displays the surfacing error (distance between the desired waypoint and the actual surfacing location) versus the length of the trajectory, i.e., distance from $L$ to the prescribed waypoint $W_{i}$. The $R^{2}$ value for the regression line shown in Fig. 3 is 0.8155 . We note the linear trend; the longer that the glider 


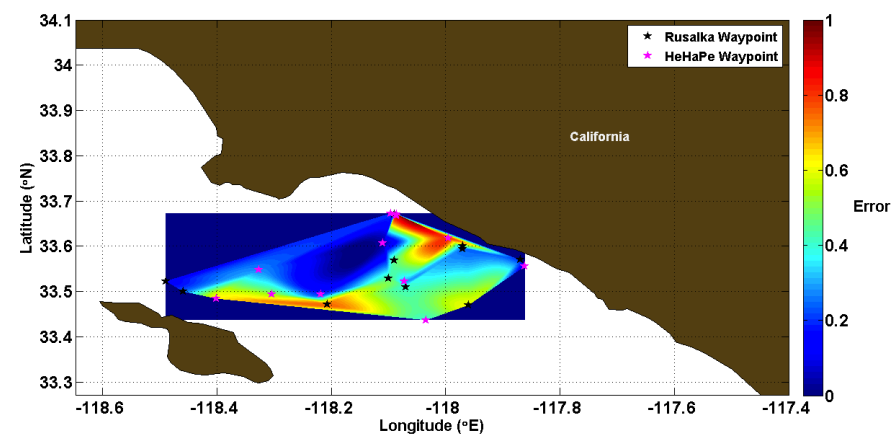

Fig. 4. A heat-map surface plot representing the normalised error between the surfacing location and the prescribed waypoint for the actual field trials.

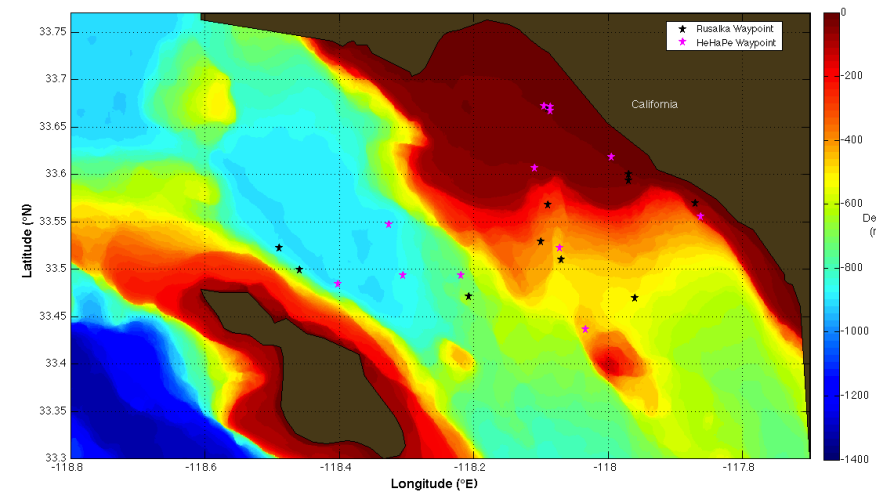

Fig. 5. A heat-map surface plot of the bathymetry for the deployment region in the SCB. Depth is taken to be zero at the surface and increase positively from red to blue.

dead-reckons underwater, the greater the surfacing error. There are a few outliers for the longer distances travelled, however we see a reasonable fit for the trajectories of length $<5 \mathrm{~km}$, which is normal for the standard missions being executed. As the surfacing error depends on the distance that the vehicle travelled underwater, we normalise it for an equivalent comparison across all 34 missions. The normalised error is a non-dimensional error, computed by the formula in Eq.(1).

$$
\text { Error }_{\text {Normalised }}=\frac{d_{g}\left(S, W_{i}\right)}{d_{g}\left(L, W_{i}\right)}
$$

Here $d_{g}(x, y)$ is the geographical distance between $x$ and $y$. Figure 4 displays a heat-map surface plot of this normalised surfacing error within the deployment region for each of the 34 actual surfacing instances. Regions in blue indicate small error, while red regions are indicative of large error. The stars represent the waypoints that the two gliders were trying to achieve. The surface is computed using a cubic interpolation of the 34 data points.

To examine the errors presented in Fig. 4, we additionally plot the prescribed waypoints on a heat-map surface plot of the bathymetry for the deployment region in Fig. 5. Red regions are area of shallower water, with blue representing deeper water. Depth is zero at the surface an increases positively. The bathymetry data were provided by [40]. We remark that the largest errors occur near shore and in the shallow (shelf) portion of the region. These large errors are attributed to two factors:

1) Regional forcing drives upwelling across the shelf and creates strong and complex currents in the shallower shelf region.

2) The northernmost prescribed waypoints are spaced less than one $\mathrm{km}$ apart, and are utilised as a holding pattern. Given that the glider must travel by executing an integer number of yos, overshooting the waypoint is more likely given the short baseline distance. This increases the normalised error for the waypoints that are in close proximity geographically.

\section{B. SIMULATIONS}

Through previous research efforts, we have developed a simulation tool for glider missions in the SCB that incorporates the ROMS predictions of the 3D velocity field [26], [27]. We fuse the measurements from the sensors (either actual or simulated) into an Unscented Kalman Filter (UKF) to estimate the position, attitude and velocity of the vehicle over time [41]. The UKF is a Bayesian filtering algorithm which employs a statistical local linearisation procedure to propagate and update the system state. For nonlinear systems, this approach typically produces significantly more accurate estimates than the analytic local linearisation employed by the well-known extended Kalman filter (EKF) [42]. Our $10 \times 1$ state vector is

$$
\mathbf{x}(t)=\left[\begin{array}{lll}
\left(\mathbf{p}^{W}(t)\right)^{T} & \left(\bar{q}_{B}^{W}(t)\right)^{T} \quad\left(\mathbf{v}^{B}(t)\right)^{T}
\end{array}\right]^{T},
$$

where $\mathbf{p}^{W}(t)$ is the position of the glider in the world (UTM) frame, $\bar{q}_{B}^{W}(t)$ is the unit quaternion that defines the attitude of the glider body relative to the world frame, and $\mathbf{v}^{B}(t)$ is the velocity of the glider in the body frame. This simple kinematic model is sufficient for this application of long-range planning. A primary motivation for our choice of the UKF is its performance with a more sophisticated (and nonlinear) dynamic model of the glider, which we are exploring in a parallel effort.

For our simulation, we assume that the glider follows a nominal linear sawtooth trajectory, and that the vehicle angular rotation rate and linear acceleration are driven by white, zero-mean Gaussian noise processes represented by the vectors $\eta_{q}(t)$ and $\eta_{v}(t)$, with covariance matrices $\mathbf{Q}_{q}$ and $\mathbf{Q}_{v}$ respectively. The system state evolves in continuous time according to

$$
\begin{aligned}
\dot{\mathbf{p}}^{W}(t) & =\mathbf{C}\left(\bar{q}_{B}^{W}(t)\right) \mathbf{v}^{B}(t), \\
\dot{\bar{q}}_{B}^{W}(t) & =\frac{1}{2} \Omega\left(\eta_{q}(t)\right) \bar{q}_{B}^{W}(t), \\
\dot{\mathbf{v}}^{B}(t) & =\eta_{v}(t),
\end{aligned}
$$

where $\mathbf{C}\left(\bar{q}_{B}^{W}(t)\right)$ is the direction cosine matrix corresponding to the unit quaternion $\bar{q}_{B}^{W}(t)$, and $\Omega\left(\eta_{q}(t)\right)$ is the quaternion kinematic matrix, relating the rate of change of the orientation quaternion to the body frame angular velocity [43].

The effects of the currents on the glider are incorporated as a concatenation of the contributions from the velocity of 


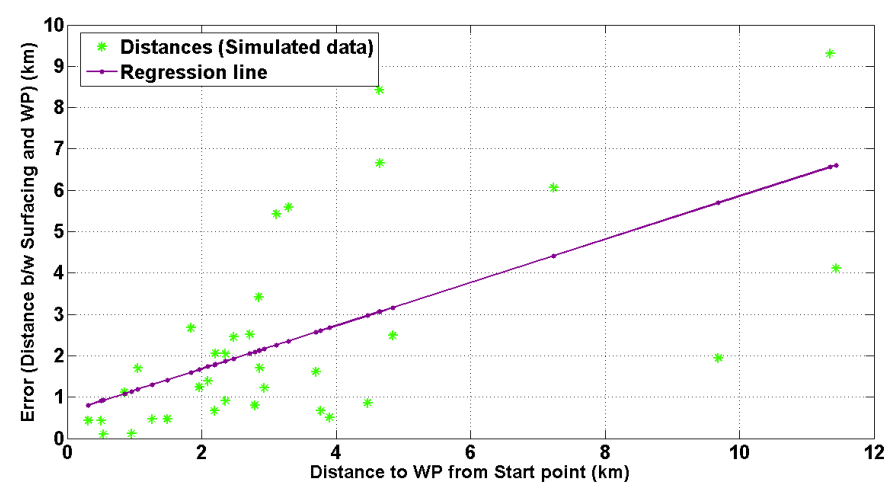

Fig. 6. A plot of the absolute surfacing error (in $\mathrm{km}$ ) versus the length of the prescribed trajectory for the simulated trials.

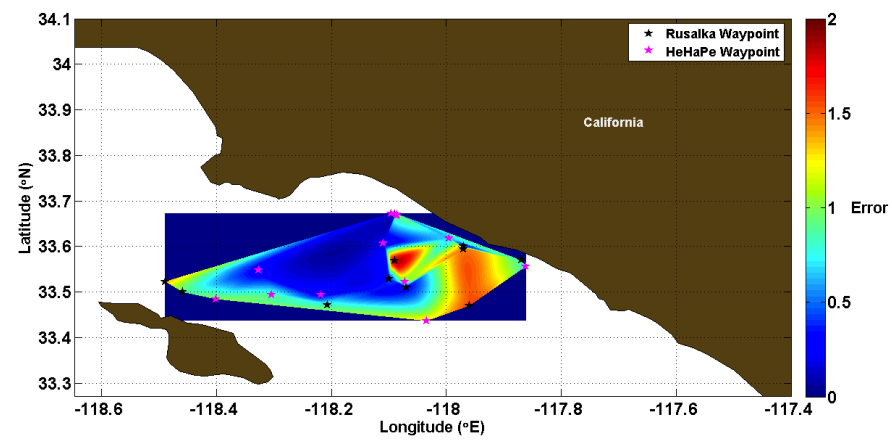

Fig. 7. A heat-map surface plot representing the normalised error between the surfacing location and the prescribed waypoint for the simulated trials.

the glider in the water column, and the velocity of the water column itself (i.e., the current). The modified process model for the glider position is then

$$
\dot{\mathbf{p}}^{W}(t)=\mathbf{C}\left(\bar{q}_{B}^{W}(t)\right) \mathbf{v}^{B}(t)+\mathbf{v}_{\mathrm{ROMS}}(t),
$$

where $\mathbf{v}_{\text {ROMS }}(t)$ is the predicted water current velocity, found by spatiotemporally interpolating the ROMS prediction.

We utilised the UKF to re-execute all of the 34 missions using the ocean current predictions from ROMS for the respective day and time of actual execution. Figure 6 displays the surfacing error versus the length of the trajectory for these simulations. The $R^{2}$ value for the displayed regression line in Fig. 6 is 0.6059. The linear trend is not as evident in Fig. 6 as previously seen in Fig. 3, with much more dispersion in the shorter-length missions. Figure 7 displays a heat-map surface plot of this normalised surfacing error within the deployment region for each of the 34 simulated surfacing instances. Regions in blue indicate small error, while red regions are indicative of large error. A normalised error of value $>1$ indicates a surfacing error greater than the length of the prescribed trajectory. The stars represent the waypoints that the two gliders were trying to achieve. The surface is computed using a cubic interpolation of the 34 data points. Here, the largest errors are seen directly along the shelf break; a region where the bathymetry changes rapidly as you move towards shore. Note that the scale is different from Fig. 4, with the error along the shelf break $>1$; the surfacing error was larger than the actual length of the

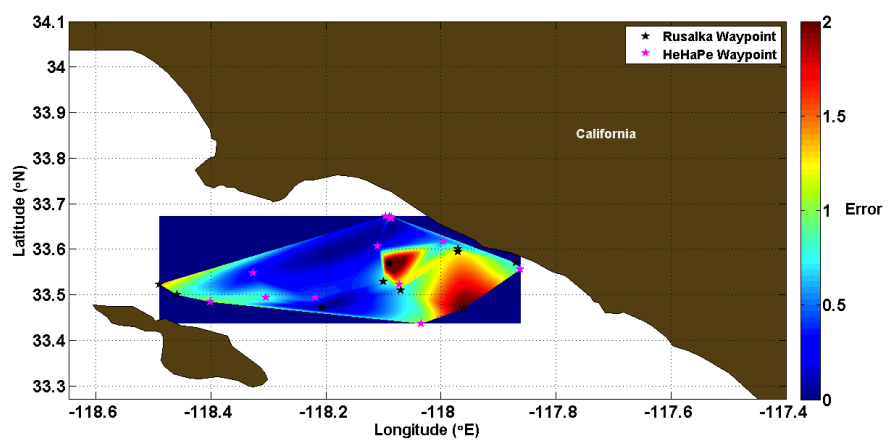

Fig. 9. A heat-map surface representing the normalised error between the actual surfacing location and the simulated surfacing for the 34 examined trials.

prescribed trajectory. It is understandable that a model would have trouble predicting currents in this complex region. Additionally, the method of spatiotemporal interpolation of the discrete predictions may result in a different flow field through this region than actually exists, causing the large errors in the simulation that do not appear in the actual field experiments.

\section{COMPARISON}

In this section, we compare the results of the surfacing locations from the field trials to those of the simulation runs, and provide a qualitative measure on the utility of ROMS predictions for path planning for autonomous gliders.

First, we examine the correlation between the surfacing locations. In Figs. 8(a) and 8(b) we present plots of the simulated surface location versus the actual surfacing location for latitude and longitude, respectively. Both plots show a distinct linear relationship between the two errors. The $R^{2}$ values for the linear regressions are 0.9785 and 0.9878 for latitude and longitude, respectively.

Figure 9 shows a heat-map surface of the error between the actual and simulated surfacings, normalised using Eq. (1) to account for the different lengths of each trajectory, for each of the 34 missions examined here. Regions in blue indicate small error, while red regions are indicative of large error. The stars represent the waypoints that the vehicles were trying to achieve. The surface is computed with a cubic interpolation of the 34 data points. Here, the areas in blue indicate a good correlation between the ocean model and actual currents experienced during field trials, while the red areas indicate regions of significant difference. There is a clear discrepancy along the shelf break, which is understandable and explained previously. However, the other region displaying large discrepancies between the model and actuality occurs in a relatively homogeneous area of bathymetry of deeper water. This error may be a result of interpolation error, or simply an excellent experiment with a poor simulation result. In either instance, further analysis must be conducted that utilises more field experiments over a greater time period.

Overall, this analysis shows a good comparison between model predictions and the results of field trial validation for 


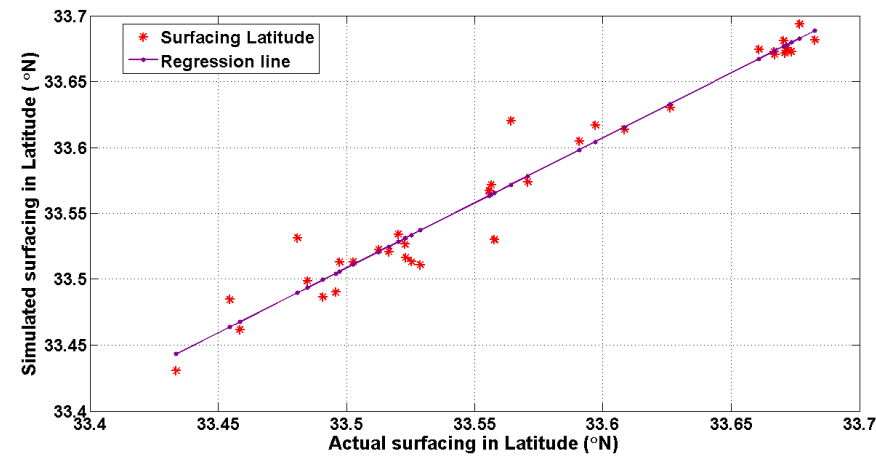

(a) Comparison in the latitudinal component.

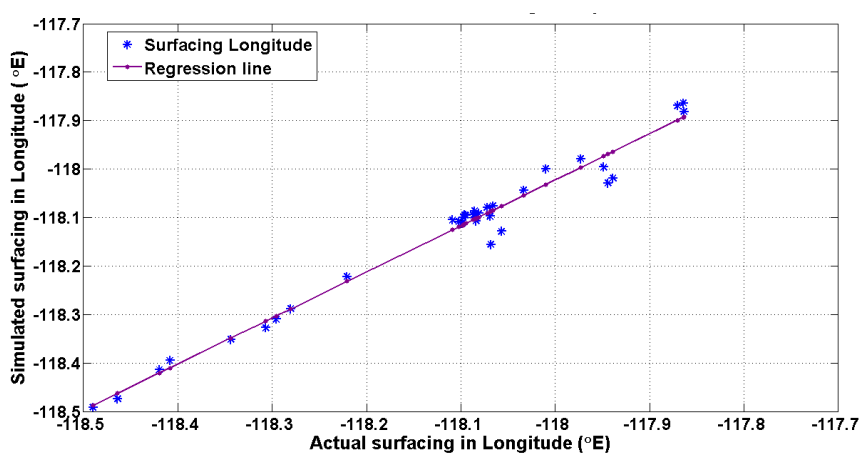

(b) Comparison in the longitudinal component.

Fig. 8. A comparison of the latitudinal 8(a) and longitudinal 8(b) components of the simulated surfacing locations versus the actual surfacing locations.

a small region in the SCB. Additionally, we have identified a key region (shelf break), where the model predictions should not be utilised for planning. These results can be incorporated into path planners by giving the regions of high discrepancy more penalty than those with good correspondence, thus increasing the utility of the given prior.

\section{CONCLUSION}

In this paper, we examined 34 prescribed path executions by autonomous gliders in a coastal region in southern California. The results of the field experiment are compared with simulations of the same missions in a UKF that integrated ROMS current predictions. We found that the model does not produce reliable predictions along the shelf break region. Additionally, it was shown that model predictions were more reliable along east-west trajectories, as compared with northsouth trajectories. In the majority of areas within the deployment region studied, ROMS provided a prediction of the ocean current that corresponded well with field experiment results. This information can be used to increase the utility of the deterministic model predictions by understanding what regions actually do have unpredictable currents.

The study presented here serves as an initial investigation into the comparison of actual ground truth with ocean model predictions. Future work will expand on this effort to include more field trials over longer time periods, as well as to compare other output variables of the model, e.g., salinity, temperature, and chlorophyll, with those measured by the vehicle(s). Additionally, we intend to examine field trials conducted during the same time period over multiple years to examine the ability of the model to predict seasonal and annual variability in the structure of the ocean currents in southern California.

\section{ACKNOWLEDGEMENTS}

This work relates to Department of the Navy Grant N62909-11-1-4046 issued by the Office of Naval Research Global. The United States Government has a royalty-free license throughout the world in all copyrightable material contained herein. We gratefully acknowledge support from the ONR Antidote MURI project, grant no. N00014-091-1031. R.N. Smith and G.S. Sukhatme were also supported in part by the NOAA MERHAB program (grant NA05NOS4781228), NSF as part of the Center for Embedded Network Sensing (CENS) (grant CCR-0120778), and NSF grant CNS-1035866. R.N. Smith was also supported in part by the Early Career Academic Recruitment and Development (ECARD) Program of the Queensland University of Technology. The authors would like to thank Carl Oberg, Matthew Ragan and Arvind Pereira for their valuable assistance with glider deployment and general operation logistics. The ROMS predictions used were computed by the Jet Propulsion Laboratory (JPL), California Institute of Technology, under a contract with the National Aeronautics and Space Administration (NASA).

\section{REFERENCES}

[1] Z. Li, Y. Chao, J. C. McWilliams, and K. Ide, "A three-dimensional variational data assimilation scheme for the regional ocean modeling system," Journal of Atmospheric and Oceanic Technology, vol. 25, pp. 2074-2090, 2008.

[2] Y. Chao, Z. Li, J. Farrara, J. C. McWilliams, J. Bellingham, X. Capet, F. Chavez, J.-K. Choi, R. Davis, J. Doyle, D. Frantaoni, P. Li, P. Marchesiello, M. Moline, J. Paduan, and S. Ramp, "Development, implementation and evaluation of a data-assimilative ocean forecasting system off the central california coast," Deep-Sea Research II, vol. 56, 2008.

[3] A. Robinson, H. Arango, A. Warn-Varnas, W. Leslie, A. Miller, P. Haley, and C. Lozano, Modern Approaches to Data Assimilation in Ocean Modeling, ch. Real-Time Regional Forecasting, pp. 377 410. Elsevier Science, 1996.

[4] A. Robinson, "Physical processes, field estimation and an approach to interdisciplinary ocean modeling," Earth-Science Reviews, vol. 40, pp. $3-54,1996$.

[5] A. Robinson, "The harvard ocean prediction system." http:// modelseas.mit. edu/HOP S/, 2012. last viewed September 2012.

[6] I. Shulman, C. R. Wu, J. K. Lewis, J. D. Paduan, L. K. Rosenfeld, J. C. Kindle, S. R. Ramp, and C. A. Collins, "High resolution modeling and data assimilation in the monterey bay area," Continental Shelf Research, vol. 22, pp. $1129-1151,2002$.

[7] I. Shulman, J. Kindle, S. Derada, S. Anderson, B. Penta, and P. Martin, "Development of hierarchy of different resolution models for study u.s. west coast california current ecosystem, in estuarine and coastal modeling," in Proceedings of $8^{\text {th }}$ International Conference on Estuarine and Coastal Modeling (M. L. Spaulding, ed.), pp. 74 - 88, 2004.

[8] I. Shulman, J. Kindle, P. Martin, S. deRada, J. Doyle, B. Penta, S. Anderson, F. Chavez, J. Paduan, and S. Ramp, "Modeling of upwelling/relaxation events with the navy coastal ocean model," Journal of Geophysical Research, vol. 112, 2007. 
[9] A. Shchepetkin and J. McWilliams, "Quasi-monotone advection schemes based on explicit locally adaptive dissipation," Monthly Weather Review, vol. 126, pp. 1541-1580, 1998.

[10] A. F. Shchepetkin and J. C. McWilliams, "The regional oceanic modeling system (ROMS): a split-explicit, free-surface, topographyfollowing-coordinate oceanic model," Ocean Modelling, vol. 9, pp. 347-404, 2005.

[11] Y. Chao, Z. Li, J. Farrara, M. Moline, O. M. E. Schofield, and S. J. Majumdar, "Synergistic applications of autonomous underwater vehicles and regional ocean modeling system in coastal ocean forecasting," Limnol. Oceanogr., vol. 53, no. 5, part 2, pp. 2251-2263, 2008.

[12] C. Lozano, A. Robinson, H. Arango, A. Gangopadhyay, Q. Sloan, P. Haley, L. Anderson, and W. Leslie, Modern Approaches to Data Assimilation in Ocean Modeling, ch. An Interdisciplinary Ocean Prediction System: Assimilation Strategies and Structured Data Models, pp. 413-452. Elsevier Science, 1996

[13] I. Shulman, C. Rowley, S. Anderson, S. DeRada, J. Kindle, P. Martin, J. Doyle, J. Cummings, S. Ramp, F. Chavez, D. Fratantoni, and R. Davis, "Impact of glider data assimilation on the Monterey Bay model," Deep-Sea Research II, vol. 56, pp. 188 - 198, 2009.

[14] B. Garau, A. Alvarez, and G. Oliver, "Path planning of autonomous underwater vehicles in current fields with complex spatial variability: an A* approach," in Proceedings of the IEEE International Conference on Robotics and Automation, (Barcelona, Spain), 2005.

[15] C. Petres, Y. Pailhas, P. Patron, Y. Petillot, J. Evans, and D. Lane, "Path planning for autonomous underwater vehicles," IEEE Transactions on Robotics, vol. 23, no. 2, pp. 331-341, 2007.

[16] D. Kruger, R. Stolkin, A. Blum, and J. Briganti, "Optimal auv path planning for extended missions in complex, fast-flowing estuarine environments," in Proceedings of the IEEE International Conference on Robotics and Automation, (Rome, Italy), 2007.

[17] J. Witt and M. Dunbabin, "Go with the flow: Optimal path planning in coastal environments," in Proceedings of the 2008 Australasian Conference on Robotics \& Automation (ACRA 2008) (J. Kim and R. Mahony, eds.), (Canberra, ACT), 2008. ISBN: 9780646506432.

[18] R. N. Smith, Y. Chao, P. P. Li, D. A. Caron, B. H. Jones, and G. S. Sukhatme, "Planning and implementing trajectories for autonomous underwater vehicles to track evolving ocean processes based on predictions from a regional ocean model," International Journal of Robotics Research, vol. 29, pp. 1475-1497, October 2010.

[19] J. Das, F. Py, T. Maughan, T. O’Reilly, M. Messi, J. Ryan, G. S. Sukhatme, and K. Rajan, "Simultaneous tracking and sampling of dynamic oceanographic features with auvs and drifters," International Journal of Robotics Research, Apr 2012.

[20] A. Garcia-Olaya, F. Py, J. Das, and K. Rajan, "An on-line utility based multi-criteria approach for sampling dynamic ocean fields," IEEE Journal of Oceanic Engineering, 2012.

[21] B. H. Jones, M. A. Noble, and T. D. Dickey, "Hydrographic and particle distributions over the palos verdes continental shelf: spatial, seasonal and daily variability," Continental Shelf Research, vol. 22, no. 6-7, pp. 945-965, 2002.

[22] A. Schnetzer, P. E. Miller, R. A. Schaffner, B. Stauffer, B. H. Jones, S. B. Weisberg, P. M. DiGiacomo, W. M. Berelson, and D. A. Caron, "Blooms of pseudo-nitzschia and domoic acid in the san pedro channela and los angeles harbor areas of the southern california bight, 2003-2004," Harmful Algae, vol. 6, no. 3, pp. 372-387, 2007.

[23] R. N. Smith, J. Das, H. Heidarsson, A. Pereira, I. Cetinić, L. Darjany, M. Ève Garneau, M. D. Howard, C. Oberg, M. Ragan, A. Schnetzer, E. Seubert, E. C. Smith, B. A. Stauffer, G. Toro-Farmer, D. A. Caron, B. H. Jones, and G. S. Sukhatme, "USC CINAPS builds bridges: Observing and monitoring the Southern California Bight," IEEE Robotics and Automation Magazine, Special Issue on Marine Robotics Systems, vol. 17, pp. 20-30, March 2010.

[24] I. Cetinic, B. H. Jones, M. A. Moline, and O. Schofield, "Resolving urban plumes using autonomous gliders in the coastal ocean," Continental Shelf Research, 2010. In review.

[25] Q. Vu, "JPL OurOcean portal." http: //ourocean.jpl.nasa. gov/, 2008. viewed January 2013.

[26] R. N. Smith, J. Kelly, Y. Chao, B. H. Jones, and G. S. Sukhatme, "Towards improvement of autonomous glider navigation accuracy through the use of regional ocean models," in Proceedings of the 29th International Conference on Offshore Mechanics and Arctic Engineering, (Shanghai, China), pp. 597-606, June 2010.

[27] R. N. Smith, J. Kelly, and G. S. Sukhatme, "Towards improving mission execution for autonomous gliders with an ocean model and kalman filter," in Proceedings of the IEEE International Conference on Robotics and Automation, (Minneapolis, MN), May 2012.

[28] M. Rixen, J. W. Book, A. Carta, V. Grandi, L. Gualdesi, R. Stoner, P. Ranelli, A. Cavanna, P. Zanasca, G. Baldasserini, A. Trangeled, C. Lewis, C. Trees, R. Grasso, S. Giannechini, A. Fabiani, D. Merani, A. Berni, M. Leonard, P. Martin, C. Rowley, M. Hulbert, A. Quaid W. Goode, R. Preller, N. Pinardi, P. Oddo, A. Guarnieri, J. Chiggiato, S. Carniel, A. Russo, M. Tudor, F. Lenartz, and L. Vandenbulcke, "Improved ocean prediction skill and reduced uncertainty in the coastal region from multi-model super-ensembles," Journal of Marine Systems, vol. 78, Supplement, no. 0, pp. S282 - S289, 2009. Coastal Processes: Challenges for Monitoring and Prediction.

[29] B. Powell, H. Arango, A. Moore, E. D. Lorenzo, R. Milliff, and D. Foley, "4dvar data assimilation in the intra-americas sea with the regional ocean modeling system (roms)," Ocean Modelling, vol. 25, no. 3 - 4, pp. 173 - 188, 2008.

[30] A. Alvarez, A. Caiti, and R. Onken, "Evolutionary path planning for autonomous underwater vehicles in a variable ocean," IEEE Journal of Oceanic Engineering, vol. 29, no. 2, pp. 418-429, 2004.

[31] M. Ono and B. C. Williams, "An efficient motion planning algorithm for stochastic dynamic systems with constraints on probability of failure," in Proceedings of the Twenty-Third AAAI Conference on Artificial Intelligence, 2008.

[32] D. Rao and S. B. Williams, "Large-scale path planning for underwater gliders in ocean currents," in Proceedings of the Australasian Conference on Robotics and Automation, (Sydney), December 2009.

[33] G. A. Hollinger, B. Englot, F. Hover, U. Mitra, and G. S. Sukhatme, "Uncertainty-driven view planning for underwater inspection," in IEEE International Conference on Robotics and Automation, (St. Paul, MN), pp. 4884-4891, May 2012.

[34] Webb Research Corporation. http://www.webbresearch. com/s locum. htm, 2008. viewed March 2011.

[35] O. Schofield, J. Kohut, D. Aragon, E. Creed, J. Graver, C. Haldman, J. Kerfoot, H. Roarty, C. Jones, D. Webb, and S. Glenn, "Slocum gliders: Robust and ready," Journal of Field Robotics, vol. 24, no. 6 , pp. 473-485, 2007.

[36] G. Griffiths, C. Jones, J. Ferguson, and N. Bose, "Undersea gliders," Journal of Ocean Technology, vol. 2, no. 2, pp. 64-75, 2007.

[37] R. E. Davis, M. Ohman, D. Rudnick, J. Sherman, and B. Hodges, "Glider surveillance of physics and biology in the southern california current system," Limnol. Oceanogr., vol. 53, no. 2, pp. 2151-2168, 2008.

[38] R. N. Smith, M. Schwager, S. L. Smith, D. Rus, and G. S. Sukhatme, "Persistent ocean monitoring with underwater gliders: Towards accurate reconstruction of dynamic ocean processes," in Proceedings of the International Conference on Robotics and Automation, (Shanghai, China), pp. 1517 - 1524, 2011.

[39] R. N. Smith, M. Schwager, S. L. Smith, B. H. Jones, D. Rus, and G. S. Sukhatme, "Persistent ocean monitoring with underwater gliders Adapting sampling resolution," Journal of Field Robotics, vol. 28, pp. 714 - 741, September/October 2011

[40] D. Divins and D. Metzger, "Ngdc coastal relief model." http: // www.ngdc.noaa.gov/mgg/coastal/coastal.html. Retrieved September 2012. Download provided by the Southern California Coastal Ocean Observing System http://sccoos.org/ data/bathy.

[41] S. J. Julier and J. K. Uhlmann, "Unscented filtering and nonlinear estimation," Proceedings of the IEEE, vol. 92, pp. 401-422, March 2004.

[42] A. Huster and S. M. Rock, "Relative position sensing by fusing monocular vision and inertial rate sensors," in Proceedings of the 11th International Conference on Advanced Robotics (ICAR'03), vol. 3 , (Coimbra, Portugal), pp. 1562-1567, July 2003.

[43] B. L. Stevens and F. L. Lewis, Aircraft Control and Simulation. WileyInterscience, Second ed., October 2003. 